\title{
Perfiles Diferenciales de Usuarios de Internet, Factores de Personalidad, Rasgos Positivos, Síntomas Psicopatológicos y Satisfacción com la Vida
}

\author{
Differential Profiles of Internet Users, Personality Factors, Positive Traits, \\ Psychopathological Symptoms and Life Satisfaction
}

\author{
Alejandro Castro Solano ${ }^{1}$ y María Laura Lupano Perugini ${ }^{2}$
}

\begin{abstract}
Resumen
Esta investigación tuvo como objetivo identificar diferentes perfiles de usuarios de Internet y redes sociales, considerando variables relacionadas con el uso, aspectos sociodemográficos y psicológicos. La muestra estuvo compuesta por 1248 sujetos del área metropolitana de Buenos Aires, Argentina (620 hombres, 628 mujeres); edad promedio: 39.41 años $(D E=14.38)$. Para la recopilación de datos, esta investigación utilizó: Big Five Inventory, High Five Inventory, Symptom Checklist-90-R, Satisfaction with Life Scale y una encuesta diseñada ad-hoc para evaluar los diferentes usos que las personas hacen de Internet y, en especial de las redes sociales. Se generaron diferentes perfiles que muestran que el intenso uso de Internet especialmente de las redes sociales- generan una disminución en los niveles de satisfacción con la vida y un aumento de síntomas psicopatológicos. La extraversión, la jovialidad y la baja responsabilidad parecen ser factores determinantes en las preferencias por el uso de las redes sociales.
\end{abstract}

Palabras clave: Internet, personalidad, rasgos positivos, síntomas, satisfacción

\begin{abstract}
This research aimed to identify different profiles of Internet and Social Networks users, considering variables linked with use, sociodemographic and psychological aspects. The sample consisted of 1248 subjects from the metropolitan area of Buenos Aires, Argentina (620 men, 628 women); average age: 39.41 years $(\mathrm{SD}=14.38)$. For data collection this research used: Big Five Inventory, High Five Inventory, Symptom Checklist-90-R, Satisfaction with Life Scale, and a survey designed ad-hoc to assess peoples' different uses of the Internet and of social networks in particular. Different profiles were generated which show that intense Internet use of social networks in particular generate a decrease in life satisfaction levels and an increase in psychopathological symptoms. Extraversion, joviality and low responsibility seem to be determining factors in preferences for the use of social networks.
\end{abstract}

Keywords: internet, personality, positive traits, symptoms, satisfaction

El presente proyecto fue realizado mediante los subsidios PIP CONICET 11220150100381CO "Hacia una aproximación émica de la psicología positiva. Los rasgos positivos como predictores del funcionamiento óptimo" y con subsidio UBACyT, 20020150100037BA "La evaluación de los rasgos de personalidad positivos. Su relación con los rasgos de personalidad patológicos (DSM5) y el bienestar psicológico".

\footnotetext{
${ }^{1}$ Dr. en Psicología. Miembro de la Carrera del Investigador del CONICET (Consejo Nacional de Investigaciones Científicas y Técnicas), Buenos Aires, Argentina. Dirección: Paraguay 5337 - 5to A. Buenos Aires. Arg. Tel. (54-11) 4771-6029. Correo: a.castro@fibertel.com.ar

${ }^{2}$ Dra. en Psicología. Miembro de la Carrera del Investigador del CONICET (Consejo Nacional de Investigaciones Científicas y Técnicas), Buenos Aires, Argentina. Correo: mllupano@hotmail.com
} 


\section{Introducción}

Hoy en día resultaría imposible imaginar un mundo sin Internet. En las últimas décadas su uso ha crecido de forma exponencial y ha influenciado el estilo de vida de las personas, los modos de comunicarse, de establecer relaciones y de buscar información (Huang, 2010), trayendo consigo algunos riesgos (Diomidous et al., 2016; Marques, Marques Pinto, \& Alvarez, 2016). Una de las áreas en las que Internet ha introducido mayores cambios ha sido en nuestra vida social (AmichaiHamburger, Wainapel, \& Fox, 2002; Hamburger \& Ben-Artzi, 2000).

En los últimos años se ha registrado un aumento en la cantidad de tiempo que la gente emplea navegando en internet, esto ha motivado numerosas investigaciones acerca de los efectos psicológicos que esto genera. La literatura internacional señala que la cantidad promedio de tiempo que la gente emplea solamente en el uso de redes sociales como Facebook varía de 30 minutos a más de 2 horas diarias (Kalpidou, Costin, \& Morris, 2011; Tsitsika et al., 2014). Un estudio reciente realizado en Argentina, mostró que el $87.6 \%$ de las personas encuestadas dijo estar todos los días conectado a algún sitio de internet siendo el promedio de horas de conexión diaria igual a 7.6 horas (Delfino, Sosa, \& Zubieta, 2017).

En general, uno de los usos más frecuentes de Internet es con fines de comunicación interpersonal, sobre todo desde la creación de Facebook en 2004 y el creciente uso de smarthphones, que la han potenciado como una herramienta comunicativa a través de las redes sociales (e.g., Facebook, Twitter, Instagram), blogs y mensajes instantáneos como Whatssap. Sin embargo, existe una multiplicidad de otros usos (e.g., jugar on line, buscar información, leer noticias, realizar compras, etc.), por lo que algunos autores consideran que el uso diferencial puede ser una variable moderadora en los efectos (Wilson, Gosling, \& Graham, 2012).

La investigación psicológica sobre los efectos positivos y negativos de los diferentes usos de Internet y la contribución de ciertas variables individuales, data de finales de los años 90. El trabajo original de Kraut et al. (1998) es una de las primeras investigaciones empíricas sobre los efectos de los usos de Internet en una población que no había tenido contacto con este tipo de tecnología. Los autores habían concluido que el uso continuado de Internet reducía el bienestar psicológico de los participantes y aumentaba su depresión y aislamiento. Denominaron a este efecto Internet Paradox, ya que era una tecnología principalmente diseñada para favorecer la comunicación interpersonal y finalmente su uso reiterado la reducía. Un estudio posterior de seguimiento de estos participantes señalaba que este efecto se disipaba (Kraut et al., 2002) y que el uso de Internet tenía diferentes efectos en función de los rasgos de personalidad de los usuarios. El estudio mostraba que esta variable diferencial (personalidad) moderaba el efecto de la relación y que los extrovertidos aumentaban su bienestar y autoestima y disminuían su aislamiento y emociones negativas. En cambio, aquellos introvertidos que enfatizaban el uso obtenían resultados opuestos. Lo hallado daba sustento a la hipótesis que denominaron "Riche gets richer" que indicaba que los extrovertidos se beneficiaban del alto uso de Internet, en tanto que los introvertidos empeoraban su condición.

En virtud de lo expuesto, muchos estudios han abordado la relación entre el uso de Internet y determinados rasgos de personalidad, principalmente considerando el modelo de los cincos factores o Big Five (Goldberg et al., 2006). En general, los trabajos muestran que los sujetos extravertidos y menos responsables suelen hacer un mayor uso de las redes sociales (AmichaiHamburger \& Vinitzky, 2010; Blackwell, Leaman, Tramposch, Osborne, \& Liss, 2017). Por el contrario, las personas con alto nivel de responsabilidad las consideran una pérdida de tiempo por su alto poder distractor (Ryan \& Xenos, 2011). Además, aquellos con un elevado nivel de responsabilidad y/o neuroticismo tienen un alto control sobre la información personal que publican (Seidman, 2013). En general el rasgo neuroticismo se asocia con un uso problemático de internet (Kayiş et al., 2016). Algo similar ocurre con las personas que se caracterizan por el rasgo agradabilidad ya que, al estar interesados en caer bien, manejan su autopresentación hablando de sí mismos en términos demasiado positivos (Fullwood, Nicholls, \& Makichi, 2014). Por último, los antecedentes muestran resultados 
contradictorios respecto del rasgo apertura a la experiencia. En general, se lo asocia con perfiles de usuarios que emplean diferentes características de las redes, posteo continuo de fotos y uso de internet con fines de búsqueda de información, dando cuenta de la versatilidad de intereses (Amichai-Hamburger \& Vinitzky, 2010). Algunos autores sostienen que los resultados de estos estudios son mixtos e inconclusos por lo que se debe continuar profundizándolos (Gilbert \& Barton, 2013).

En el presente estudio se analizan, además de los rasgos basados en el Big Five, una serie de rasgos positivos (erudición, paz, jovialidad, honestidad, y tenacidad) que fueron obtenidos mediante un método inductivo -basado en el enfoque léxico- en el que se analizaron palabras que las personas emplean para definir características positivas (morales y no morales). La novedad y aporte de este modelo denominado Modelo de los Cinco Altos (MCA) o High Five Model, (HFM) es que estos rasgos permiten predecir el bienestar por sobre lo explicado por los rasgos normales del Big Five (Cosentino \& Castro Solano, 2017).

Por otro lado, muchas investigaciones han analizado la relación entre el uso de internet y los niveles de bienestar psicológico y de satisfacción vital de los usuarios. En este punto, la naturaleza de esta asociación también es poco clara ya que algunos estudios muestran contribuciones beneficiosas y otros apuntan a los efectos perjudiciales. De acuerdo con Nie, Sousa-Poza y Nimrod (2016), estas diferencias pueden deberse al empleo de diferentes medidas de bienestar, tipo de población estudiada, diferentes focos en el análisis (e.g., Uso/ no uso vs. Usos específicos), diversas formas de considerar el tiempo empleado en el uso (e.g., cantidad de horas diarias, semanales, etc.), entre otros factores. A pesar de la disparidad en los resultados, en general los estudios coinciden en que los efectos más nocivos se suelen dar en aquellos que hacen un uso pasivo de Internet, es decir en aquellos que solo ven o buscan publicaciones pero que no generan intercambio con otros (e.g., Burke, Marlow, \& Lento, 2010; Deters \& Mehl, 2013; Kalpidou et al., 2011; Krasnova, Wenninger, Widjaja, \& Buxmann, 2013; Verduyn, et al., 2015). También los antecedentes sugieren que las cohortes más jóvenes son más vulnerables a los efectos potencialmente negativos del uso de Internet (e.g., Anderson, Steen, \& Stavropoulos, 2016; GálvezNieto, Vera-Bachman, Cerda, \& Díaz, 2016; Nie, et al, 2016).

En continuidad con lo expuesto, los estudios que reportan efectos perjudiciales sostienen que la comunicación virtual no puede reemplazar la comunicación cara a cara generando en determinados perfiles de personalidad aspiraciones sociales difíciles de alcanzar lo que puede aumentar los niveles de frustración y disminuir los niveles de satisfacción con la vida (Nie, Hillygus, \& Erbring, 2002; Kim \& Lee, 2011). Las investigaciones que han analizado la relación entre el uso de internet (especialmente redes sociales) y presencia de sintomatología clínica destacan que existe asociación entre un elevado uso de internet (sobretodo, de tipo pasivo) y la presencia principalmente de sintomatología depresiva y de ansiedad social (e.g., Blease, 2015; Guedes, Nardi, Guimarães, Machado, \& King, 2016; Laconi, Tricard, \& Chabrol, 2015; Shaw, Timpano, Tran, \& Joormann, 2015).

En términos generales, la mayoría de los estudios han sido llevados a cabo en países desarrollados. Por dicha razón, resulta interesante analizar la interrelación entre las distintas variables mencionadas en cuanto al uso de internet en el contexto latinoamericano ya que algunos trabajos han analizado perfiles diferenciales de uso considerando, por ejemplo, variables sociodemográficas y culturales (e.g., Delfino et al., 2017) pero no teniendo en cuenta las variables psicológicas aquí tratadas.

En virtud de lo expuesto, es el presente estudio se busca identificar perfiles de usuarios de internet y redes sociales considerando variables asociadas al uso (horas de navegación, cantidad de fotos, estados de ánimo y videos compartidos), variables sociodemográficas (sexo, edad, estado civil) y variables psicológicas (sintomatología clínica psicopatológica, satisfacción con la vida, factores de personalidad, rasgos positivos).

\section{Método}

\section{Participantes}

Participaron 1248 sujetos (620 hombres, $49.7 \%$ y 628 mujeres, $50.3 \%$ ) que tenían en 
promedio 39.41 años $(D E=14.38)$ residentes en la Ciudad Autónoma de Buenos Aires $(n=702,68 \%)$ y el Conurbano Bonaerense $(n=330,32 \%)$. La mayoría de los participantes trabajaban $(n=992$, $79.5 \%$ ), ubicándose en las categorías de empleado $(n=708,56.7 \%)$. Por otro lado, un bajo porcentaje trabajaba por su cuenta $(n=192,15.4 \%)$ o bien se ubicaba en la categoría de patrón o empleador $(n=92, \quad 7.4 \%) .15$ participantes manifestaron trabajar (1.2\%) pero no percibían salario por ello.

En cuanto al nivel de estudios, el $46.3 \%$ $(n=578)$ refirió tener estudios universitarios o terciarios completos. Un $37.7 \% \quad(n=470)$ aludió tener estudio terciarios o universitarios $\sin$ terminar. Un $11.1 \% \quad(n=138)$ indicó tener el secundario completo. El restante $4.8 \% \quad(n=60)$ tenía secundario incompleto o escolaridad primaria completa. La mayoría de los participantes pertenecían a un nivel socioeconómico medio $(n=832 ; 66.7 \%)$ y medioalto $(n=288 ; 23.1 \%)$.

Se trata, por lo tanto, de una muestra de conveniencia, de población general urbana altamente educada y económicamente activa, de nivel socioeconómico medio a medio-alto.

\section{Materiales}

Encuesta de Usos de Internet. A los fines de este estudio se diseñó una encuesta para evaluar los diferentes usos que las personas hacen de Internet y de las redes sociales en particular. Respecto de los usos de Internet se encuestaron 12 usos típicos tanto recreativos (e.g., mirar videos, escuchar música); sociales (e.g., chatear con amigos o familia, compartir estados de ánimo en Facebook o Twitter) o netamente utilitarios (e.g., trabajar, hacer comprar on-line). La encuesta tenía un formato de respuesta Likert en el que los participantes debían indicar la frecuencia de la realización de cada una de las actividades en 5 opciones que iban de 1 (Nunca) a 5 (Siempre). Respecto de las redes sociales, en primer lugar, se preguntó si el participante era o no miembro de alguna red social y en caso afirmativo se encuestaron los 7 usos más frecuentes de las redes sociales (e.g., hacer nuevos amigos, compartir fotos, compartir estados de ánimo, mantenerse en contacto con familiares y amigos, etc.). La encuesta tenía un formato de respuesta Likert en el que los participantes debían indicar la frecuencia con la que utilizaban las redes sociales para cada una de las actividades encuestadas en 5 opciones que iban de 1 (Nunca) a 5 (Siempre). Asimismo, se encuestó las horas de navegación diarias en Internet y la cantidad de horas diarias que los participantes usaban las redes sociales.

Además, se emplearon los siguientes instrumentos de evaluación:

Big Five Inventory (BFI) (John, Donahue, \& Kentle, 1991; adaptación argentina Castro Solano \& Casullo, 2001): Consiste en un instrumento de 44 ítems que evalúa los cinco grandes rasgos de personalidad (extraversión, agradabilidad, responsabilidad, neuroticismo, apertura a la experiencia). La prueba es un derivado de una prueba de adjetivos de personalidad, convertidos en frases cortas para facilitar la comprensión de los elementos del test. El autor de la técnica demostró su validez y fiabilidad en grupos de población general adulta norteamericana. Esos estudios verificaron la validez concurrente con otros instrumentos reconocidos que evalúan personalidad. Estudios realizados en Argentina verificaron la validez factorial de los instrumentos para población adolescente, población adulta no consultante y población militar (Castro Solano, 2005; Castro Solano \& Casullo, 2001). En todos los casos se obtuvo un modelo de cinco factores que explicaban alrededor del $50 \%$ de la variancia de las puntuaciones. Para esta muestra se obtuvieron valores de consistencia interna adecuados: extraversión: .76; agradabilidad: .79; responsabilidad: .82; neuroticismo: .74; apertura a la experiencia: 69 .

High Five Inventory (HFI) (Cosentino \& Castro Solano, 2017): El HFI es un instrumento de medición basado en el Modelo de los Cinco Altos (MCA; High Five Model, HFM, por sus siglas en inglés) que establece cinco rasgos positivos de la personalidad: erudición, paz, jovialidad, honestidad, y tenacidad, llamados factores altos. Este instrumento fue obtenido a través de un procedimiento inductivo que partió del punto de vista de las personas comunes sobre las características positivas (morales o no morales). La escala está compuesta por 23 ítems. Se le pide al participante que responda a los ítems (p. ej., tengo paciencia) utilizando una escala que va 
de 1 (Nunca) a 7 (Siempre). A mayor puntuación de cada subescala, más elevado factor alto. El HFI presentó las siguientes evidencias de validez: convergente y divergente con relación a la clasificación Values in Action (VIA) de Peterson y Seligman (2004), e incremental por sobre los factores y facetas del Modelo de los Cinco Grandes en la predicción de las tres dimensiones del modelo Salud Mental Positiva. Además, el HFI presentó un buen ajuste a los datos tanto de la muestra de generación (e.g., CFI=.968) como de la muestra de confirmación (e.g., CFI=.963). La confiabilidad alfa y omega para cada factor es superior a .80. La consistencia interna de los factores es adecuada (jovialidad $=.90$; honestidad $=.88$; tenacidad $=.86$; erudición $=.85 ;$ paz $=.85$ ).

Symptom Checklist-90-R (SCL-90-R) (Derogatis, 1977; adaptación argentina Casullo \& Castro Solano, 1999): El SCL-90-R evalúa sintomatología específica experimentada durante la última semana en nueve dimensiones (somatización, obsesiones y compulsiones, sensitividad interpersonal, depresión, ansiedad, hostilidad, ansiedad fóbica, ideación paranoide y psicoticismo). Asimismo, se incluyen nueve ítems críticos y tres índices (índice de severidad global, índice positivo de malestar, y total de síntomas positivos). El SCL-90-R está compuesto por 90 ítems que se responden en una escala de formato Likert en 5 puntos que va de 1 (nada) a 5 (mucho). Los estudios argentinos demostraron las propiedades psicométricas del instrumento (Casullo \& Castro Solano, 1999). Para este estudio se utilizó el índice de severidad global como indicador general de sintomatología.

Escala de satisfacción con la vida (SWLS). (Diener, Emmons, Larsen \& Griffin, 1985; adaptación argentina Castro Solano, 2000): Se utilizó una versión adaptada al idioma español para uso con población argentina (Castro Solano, 2000) de la Satisfaction with Life Scale de Diener y colaboradores (1985). Es una escala de cinco ítems con formato de respuesta Likert en 7 categorías y examina el grado de satisfacción global con la vida. La escala es utilizada internacionalmente para la evaluación del bienestar en tanto componente cognitivo de la satisfacción, por ejemplo: "En la mayoría de los sentidos, mi vida está cerca de mis ideales".
Diferentes estudios empíricos han demostrado su validez y confiabilidad (Diener et al., 1985; Diener \& Larsen, 1991; Larsen, Diener, \& Emmons, 1985). Se obtiene una puntuación promedio que indica el grado de satisfacción percibida por el evaluado. Un estudio realizado sobre una muestra de argentinos adultos reveló una fiabilidad igual a .84 (Cosentino, 2011). La fiabilidad evaluada mediante el coeficiente Alpha de Cronbach para la muestra de los participantes de este estudio fue igual a .85 .

\section{Procedimiento}

Los datos fueron recolectados por alumnos que se encontraban realizando una práctica de investigación en una universidad privada de la ciudad de Buenos Aires. Los participantes fueron voluntarios y no recibieron retribución alguna por su colaboración. Además, el cuadernillo que contenía las encuestas presentaba en su portada una introducción en la que se solicitaba el consentimiento del participante, se aseguraba el anonimato de los datos y su uso exclusivo para investigación. La recolección y carga de datos fue supervisada por un docente investigador.

Para la carga y análisis de los datos se utilizaron los programas SPPS 17.0

\section{Resultados}

\section{Análisis de datos}

Con el objetivo de identificar los perfiles de usuarios de Internet y establecer su relación con las variables de interés consideradas se realizó primero un análisis factorial exploratorio de los diferentes usos de Internet y de los diferentes usos de las redes sociales con el objetivo de reducir los datos. Se obtuvieron dos factores de usos genéricos de Internet (Uso Utilitario y Uso Social) y un solo factor para el uso de redes sociales. A continuación, se obtuvieron correlaciones bivariadas entre los factores y las puntuaciones de las variables: síntomas, rasgos positivos, factores de personalidad y satisfacción con la vida.

El paso siguiente fue obtener conglomerados tanto de los usos de Internet como de las redes sociales. Para ello se llevaron a cabo dos análisis de clústeres de perfiles. Se siguió la metodología del clúster bietápico. Esta herramienta de exploración 
Tabla 1. Usos de Internet $(\mathrm{n}=1248)$

\begin{tabular}{lcc}
\hline & $\begin{array}{c}\text { Factor 1 } \\
\text { Uso Social }\end{array}$ & $\begin{array}{c}\text { Factor 2 } \\
\text { Uso Utilitario }\end{array}$ \\
\hline Varianza & $42 \%$ & $14 \%$ \\
\hline Compartir fotos y videos en los que aparezco & .86 & .81 \\
Sacar y enviar fotos en las redes sociales & .79 & .64 \\
Compartir estados de ánimo en redes sociales & .47 & .77 \\
Hacer nuevos amigos & & .76 \\
Chatear con amigos o familiares & & .75 \\
Enviar Correos Electrónicos & & .65 \\
Leer Noticias & & .61 \\
Trabajar & & .58 \\
Ver videos & & \\
Hacer compras on-line & & \\
Escuchar música & & \\
\hline
\end{tabular}

identifica las agrupaciones naturales de un conjunto de datos y permite identificar el número óptimo de los conglomerados y la calidad del ajuste de la agrupación obtenida (Rubio-Hurtado, \& Vilà-Baños, 2017; Pérez, 2011). Se utilizó la medida de distancia de log-verosimilitud y el criterio de conglomeración bayesiano de Schwartz (BIC). Por último, se realizaron análisis de la variancia con el propósito de verificar diferencias individuales en las variables consideradas para cada uno de los perfiles.

\section{Reducción de los datos}

El primer paso fue reducir los datos de la encuesta de usos de Internet y de uso de redes sociales. En primer lugar, se verificó la adecuación de los datos para este tipo de análisis (Para el uso de Internet: Test de esfericidad de Bartlett $=5922.58, g l=55, p<.0001$; Índice Kaiser Meyer Olkin $=.83$; y para el uso de redes sociales: Test de esfericidad de Bartlett $=1987.37, g l=21$, $p<.0001$; Índice Kaiser Meyer Olkin =.81). Se extrajeron los factores mediante el método de componentes principales y la solución fue rotada mediante el método Varimax (Ver datos en Tabla 1). Para el uso de Internet se obtuvieron dos factores que explicaban el $56 \%$ de la varianza. El primer factor ( $42 \%$ de la variancia) se identificó como Uso social de internet (e.g., chatear con amigos o familiares, hacer nuevos amigos, compartir estados de ánimo en las redes sociales, sacar y enviar fotos, y el segundo factor $(14 \%$ de la variancia) se denominó Uso utilitario de Internet (e.g., mirar videos, leer noticias, hacer compras, enviar correos electrónicos). En cuanto al uso de redes sociales, se obtuvo un solo factor que explicaba el $44 \%$ de la variancia. Este factor incorporaba todos los usos encuestados de las redes sociales (e.g., hacer nuevos amigos, compartir fotos, obtener comentarios de contactos, compartir estados de ánimo, etc.).

\section{Correlaciones bivariadas}

Como paso siguiente se obtuvieron las correlaciones bivariadas entre los factores encontrados y las variables de interés.

Para el uso de Internet, se encontraron dos correlaciones significativas entre el factor Utilitario y el rasgo positivo Tenacidad ( $r=.07$, $p<.05)$ y el rasgo de personalidad Apertura ( $r=-.13$, $p<.01)$. Las personas que hacen un uso de Internet mayormente por razones prácticas o utilitarias tienden a tener menor bienestar social, menor apertura a la experiencia y mayor tenacidad. Si bien las correlaciones obtenidas son todas significativas, la significación práctica es limitada ya que el tamaño del efecto de estas correlaciones es pequeño $(r<.30)$ (Cohen, 1992).

En cuanto al factor Social de usos de Internet se obtuvieron seis correlaciones significativas: con los síntomas psicopatológicos $(\mathrm{r}=.14, p<.01)$; con los rasgos de personalidad Agradabilidad ( $\mathrm{r}=$ -.06, $p<.05)$, Responsabilidad ( $\mathrm{r}=-.10, p<.01)$ y Neuroticismo $(\mathrm{r}=.06, p<.05)$ y con los rasgos positivos Jovialidad $(\mathrm{r}=.17, p<.01)$ y Paz ( $\mathrm{r}=-.06$, $p<.05)$. A mayor uso de Internet con objetivos netamente sociales las personas tienden a tener más síntomas, más Neuroticismo, menos Agradabilidad, menos Responsabilidad, más Jovialidad y menos Paz. Si bien las correlaciones obtenidas son todas significativas, la significación práctica es limitada ya que el tamaño del efecto de estas correlaciones es pequeño $(r<.30)$.

Para el uso de Redes Sociales se encontraron 
cuatro correlaciones significativas: con los síntomas psicopatológicos $(r=.05, p<.05)$; con los rasgos de personalidad Extraversión ( $r=.06$, $p<.05)$ y Responsabilidad ( $r=-.07, p<.01)$ y con los rasgos positivos Erudición $(r=-.07, p<.01)$ y Jovialidad ( $r=.14, p<.01)$. En la medida en la que se usan más las redes sociales las personas tienden a registrar mayores síntomas psicopatológicos, más Extraversión, menos Responsabilidad, menos Erudición y más Jovialidad. Si bien las correlaciones obtenidas son todas significativas, la significación práctica es limitada ya que el tamaño del efecto de estas correlaciones es pequeño $(r<.30)$.

\section{Análisis de Conglomerados}

El paso siguiente fue obtener conglomerados tanto de los usos de Internet como de las redes sociales. Para ello se llevaron a cabo dos análisis de clústeres de perfiles empleando la metodología del clúster bietápico. Se utilizó la medida de distancia de log-verosimilitud y el criterio de conglomeración bayesiano de Schwartz (BIC).

Para el análisis de clústeres de uso de Internet se incluyeron los dos factores obtenidos de usos (Uso Utilitario y Uso Social) y las horas de navegación diarias. Se obtuvieron dos conglomerados, cuya calidad de ajuste fue buena $(>.5)$. El primer conglomerado estaba integrado por el $57 \%$ de los casos $(n=711)$ y el segundo por el $43 \%$ restante $(n=537)$. La primera agrupación señalaba un alto uso de internet, con más de 4 horas por día de navegación en Internet repartidas en ambos usos (utilitario y social). La segunda agrupación identificaba un uso promedio o bajo de Internet con menos de dos horas por día también en ambos usos.

En cuanto a la composición de cada clúster no había diferencias en virtud del sexo ( $\chi 2$ (1, $N=1248)=.88, n s$.) y existían diferencias en cuanto al estado civil $(\chi 2(5, N=1248)=147.38, p<.001)$ ya que el clúster de alto uso de internet estaba integrado por personas mayormente solteras y que estaban estudiando actualmente ( $\chi 2$ (1, $N=1248)=67.84, p<.001)$. En cuanto a las edades se encontraron diferencias entre ambas agrupaciones $(t(1246)=15.18, p=.001)$. El clúster de alto uso de internet estaba integrado por personas más jóvenes en promedio $(M=34,47$, $D S=12,53)$ comparado con la agrupación de bajo uso de internet que estaba integrado por personas 11 años mayores en promedio $(M=45.94$, $D S=14.08$ ).

Para el análisis de clústeres de uso de redes sociales se incluyó el factor único de uso de redes sociales, la cantidad de horas de navegación en redes sociales y la cantidad de fotos, estados de ánimo y videos compartidos semanalmente en las redes sociales. El primer conglomerado estaba integrado por el $79 \%$ de los casos $(n=868)$ y el segundo por el $21 \% \quad(n=234)$. La primera agrupación señala un uso promedio de redes sociales, con una cantidad de horas diarias menor a dos y con menos de 5 videos, fotos y/o estados de ánimo compartido semanalmente. La segunda agrupación señala un alto uso de las redes sociales. Este grupo utilizaba más de dos horas por día las redes sociales. Una parte importante de este grupo utilizaba las redes sociales más de 6 horas diarias. En términos generales este grupo compartía una gran cantidad de fotos y estados de ánimo semanalmente.

En cuanto a la composición de las variables sociodemográficas, no existían diferencias según sexo $(\chi 2(1, N=1102)=3.10, n s$. $)$, ni en cuanto a la edad $t(1246)=15.18, p=.001)$, o si estudiaban o no $\chi 2(1, N=1102)=.25, n s)$. La agrupación de alto uso estaba integrada mayormente por personas solteras $\quad\left(\chi^{2} \quad(1, \quad N=1102)=14.66, \quad p<.01\right)$ comparadas con la de uso promedio de redes sociales.

\section{Perfiles diferenciales de uso de Internet y variables psicológicas}

Como paso siguiente se identificaron las diferencias individuales entre los perfiles de usuario de Internet y las variables psicológicas consideradas en este estudio.

En cuanto a sintomatología, no se encontraron diferencias en ambos perfiles de usuarios ( $F$ (1, $1246)=2.22, n s$.). En relación a la satisfacción con la vida se observó una diferencia estadísticamente significativa a favor del grupo de uso promedio o bajo de Internet, que registraba mayor satisfacción comparado con el grupo del alto uso de Internet $(F(1,1246)=5.08, p<.05)$.

En relación a los rasgos de personalidad se realizó un MANOVA en el que se incluyeron como variables dependientes los cinco rasgos de la personalidad y como variable independiente el 
pertenecer a una agrupación o a la otra. Se obtuvieron diferencias estadísticamente significativas entre los vectores de medias de ambos grupos (Lambda de Wilks=.98, $F(5,1242)$ $=4.52, p<.001, \eta 2=.018)$. Luego se realizaron contrastes univariados. Se encontraron diferencias entre ambos grupos para los rasgos Extraversión $(F(1,1246)=7.31, p<.01, \eta 2=.06)$, Agradabilidad $(F(1,1246)=4.17, p<.05, \eta 2=.06)$ y apertura $(F$ $(1,1246)=3.81, p<.05, \eta 2=.03)$. El grupo que utilizaba más internet resultó más extrovertido, más abierto a la experiencia y con menor Agradabilidad comparado con el grupo que hace un uso promedio o bajo de la internet.

En cuanto a los rasgos positivos se siguió un procedimiento similar al comentado para los rasgos de personalidad. Se obtuvieron diferencias estadísticamente significativas entre los vectores de medias de ambos grupos (Lambda de Wilks $=.98, \quad F(5,1242)=5.72, p<.01, \eta 2=.02)$. Los contrastes univariados arrojaron diferencias para el rasgo positivo de Jovialidad ( $F$ (1, 1246)=11.97, $p<.001, \eta 2=.10$ ) a favor del alto usuario.

\section{Perfiles diferenciales de Uso de Redes Sociales y variables de interés}

Como paso siguiente se identificaron las diferencias individuales entre los perfiles de usuario de Redes Sociales y las variables psicológicas consideradas en este estudio.

En cuanto a sintomatología, se encontraron diferencias estadísticamente significativas entre ambos perfiles de usuarios de redes $(F(1,1101)$ $=8.40, p<0.01)$. El alto usuario era el que experimentaba una mayor cantidad de sintomatología.

En relación a la satisfacción con la vida se observó una diferencia estadísticamente significativa a favor del grupo de uso promedio de redes sociales, que registraba mayor satisfacción comparado con el grupo del alto uso de Redes sociales $(F(1,1101)=3.58, p<.05)$.

En cuanto a los rasgos de personalidad se realizó un MANOVA en el que se incluyeron como variables dependientes los cinco rasgos de la personalidad y como variable independiente el pertenecer a una agrupación o a la otra. Se obtuvieron diferencias estadísticamente significativas entre los vectores de medias de ambos grupos (Lambda de Wilks $=.98, F(5,1096)$ $=3.30, p<.001, \eta 2=.015)$. Luego se realizaron contrastes univariados. Se encontraron diferencias entre ambos grupos y se obtuvo una diferencia significativa para el rasgo Responsabilidad ( $F(1$, 1096)=14.78, $p<.001, \eta 2=.013)$. El grupo que utilizaba menos las redes sociales registraba una mayor responsabilidad comparado con el grupo de alto uso de redes.

Por último, en cuanto a los rasgos positivos se siguió un procedimiento similar al comentado para los rasgos de personalidad. Se obtuvieron diferencias estadísticamente significativas entre los vectores de medias de ambos grupos (Lambda de Wilks=.97, $F(5,1096)=7.32, p<0.01, \eta 2=.03)$. Los contrastes univariados arrojaron diferencias para los rasgos positivos de Jovialidad ( $F$ (5, 1096) $=5.65, p<.01, \eta 2=.05)$, Honestidad $(F$ (5, 1096) $=11.76, p<.001, \eta 2=.010)$, y Tenacidad $(F$ $(5,1096)=8.63, p<.001, \eta 2=.08)$. El grupo que utilizaba más las redes sociales registraba más Jovialidad, menos Honestidad y menos Tenacidad comparado con el grupo que utilizaba menos las redes sociales de la Internet.

\section{Perfiles diferenciales de Uso de Redes Sociales, No Usuarios y variables de interés}

Dado que contábamos con un grupo que no utilizaba redes sociales $(n=149)$, decidimos realizar un análisis adicional. Comparamos el perfil de no usuario de redes sociales con el del usuario promedio en las variables psicológicas estudiadas.

En cuanto a sintomatología, no se encontraron diferencias estadísticamente significativas entre el usuario y el no usuario de redes $(F(1,1002)=.93$, ns.).

En cuanto a la satisfacción con la vida, se observó una diferencia estadísticamente significativa a favor del grupo no usuario de redes, que registraba mayor satisfacción vital comparado con el grupo usuario promedio de redes sociales $(F(1,1002)=5.50, p<.01)$.

En relación a los rasgos de personalidad, se realizó un MANOVA en el que se incluyeron como variables dependientes los cinco rasgos de la personalidad y como variable independiente el pertenecer a una agrupación o a la otra. No se obtuvo una diferencia estadísticamente significativa entre los vectores de medias de 
ambos grupos (Lambda de Wilks=.99, $F$ (5, 998)=.89, ns.). Los contrastes univariados tampoco arrojaban diferencias estadísticamente significativas entre los rasgos de personalidad de un grupo y el otro.

Por último, en cuanto a los rasgos positivos se siguió un procedimiento similar al comentado para los rasgos de personalidad. Se obtuvieron diferencias estadísticamente significativas entre los vectores de medias de ambos grupos (Lambda de Wilks $=.97, F(5,998)=4.29, p<0.01, \eta 2=.02)$. Los contrastes univariados arrojaron diferencias para el rasgo positivo de Jovialidad $(F(5,998)$ =4.94, $p<.05, \eta 2=.05$ ). El grupo no usuario de redes registraba menos el rasgo Jovialidad comparado con el usuario promedio de redes sociales.

\section{Discusión}

En el presente estudio se buscó identificar perfiles de usuarios de internet y redes sociales considerando variables asociadas al uso, sociodemográficas y psicológicas. En virtud de que la mayor parte de las investigaciones provienen de Estados Unidos y Europa, resulta novedoso el análisis de aspectos vinculados al uso de internet en el contexto latinoamericano.

Uno de los principales aportes se relaciona con la inclusión, entre las variables psicológicas analizadas, de los rasgos positivos de personalidad evaluados desde un modelo que ha demostrado predecir los índices de bienestar por sobre el modelo clásico del Big Five (Cosentino \& Castro Solano, 2017)

A partir de los resultados se puede concluir, en primer lugar, que la gente hace uso de internet con dos fines bien claros. Uno que refiere a un uso social (e.g., chatear con amigos) y otro a un uso utilitario (e.g., leer noticias). Tal como señalan los antecedentes (e.g., Kraut et al., 2001; Wilson et al., 2012), los efectos son distintos según el uso que se haga. Por ejemplo, quienes usan internet con fines prácticos suelen presentar menos bienestar, pero ser más tenaces; en tanto que los que hacen un uso social suelen ser menos responsables y presentar más síntomas, pero ser más joviales.

En segundo lugar, en la muestra analizada, se identificaron algunos clusters, integrados por jóvenes en su mayoría, que hacían un uso intensivo de internet y de redes sociales con una conexión de más de 4 horas diarias en las que se comparten gran cantidad de fotos y estados de ánimo. Este hallazgo se encuentra en consonancia con estudios previos que muestran una tendencia en el incremento de horas diarias de conexión (e.g., Kalpidou et al., 2011; Tsitsika et al., 2014; Delfino et al., 2017).

En tercer lugar, en este estudio se analizaron perfiles de usuarios en relación con variables psicológicas. Los resultados mostraron que el alto usuario de internet se caracteriza por percibirse con menor satisfacción vital, ser más extravertido, presentar menos agradabilidad, mayor Apertura a la experiencia y ser más jovial. Al analizar más específicamente el perfil del alto usuario de redes sociales se lo describe con mayor sintomatología clínica, menor satisfacción con la vida, menor Responsabilidad, Honestidad y Tenacidad y con mayor Jovialidad. Estos resultados se encuentran en relación con la mayor parte de los antecedentes ya que, si bien existe disparidad, en general los estudios reportan efectos negativos sobre los índices de bienestar y satisfacción y caracterizan a los usuarios como más extrovertidos y menos responsables.

Por último, al realizar la comparación con aquellos que no usan redes sociales, se halló que estos presentan un grado mayor de satisfacción vital, pero son menos joviales. A modo de síntesis, se puede decir que en parte se sigue dando la paradoja propuesta por Kraut et al. (1998) ya que un uso intenso de redes sociales que se supone que es para ampliar las interacciones sociales que se tienen offline - va acompañado de una baja en los índices de satisfacción y de un incremento en la sintomatología. Factores como la extraversión, la jovialidad y la baja responsabilidad parecen ser determinantes en las preferencias por el uso de internet con fines sociales y en especial de redes sociales.

\section{Limitaciones y futuras líneas de investigación}

En primer lugar, una de las limitaciones se relaciona con el empleo de medidas de autoinforme que pueden afectar la validez de los datos. Resultaría enriquecedor complementar con 
técnicas que permitan analizar directamente los contenidos subidos por las personas a las redes sociales a través de, por ejemplo, métodos que admitan un análisis de las palabras usadas en posteos (e.g., LIWC method; Tausczik \& Pennebaker, 2010) o de las imágenes subidas (e.g., Eftekhar, Fullwood, \& Morris, 2014). Por otro lado, y en virtud de que los estudios previos dan cuenta de que las diferencias en los efectos pueden deberse a si hace un uso pasivo o activo de las redes (e.g., Burke et al., 2010; Deters \& Mehl, 2013; entre otros), deberían incluirse ítems -en la encuesta diseñada- que permitan diferenciar a los usuarios en cuanto a ese tipo de uso y no solamente a la intensidad.

Otra limitación se relaciona con la muestra empleada ya que es altamente educada y de nivel socioeconómico medio a medio-alto. Debiera considerarse una muestra que incluya personas de menor nivel educativo y socioeconómico a fin de verificar diferencias en el uso de internet.

Por último, en este estudio no se tomó en cuenta la preferencia por el uso de determinadas redes sociales (Facebook, Instagram, Twitter, Snapchat, etc) considerando que investigaciones previas han demostrado que la preferencia puede estar dado por diferencias en los perfiles de personalidad (e.g., Hughes, Rowe, Batey, \& Lee, 2012).

En cuanto a futuras líneas de investigación, surge la necesidad de analizar el poder predictor de cada una de las variables aquí analizadas sobre los índices de bienestar de los usuarios de internet. Además, también resulta interesante analizar los efectos sobre otro tipo de variables de resultado como puede ser los índices de performance laboral en muestras de empleados $\mathrm{o}$ de rendimiento académico en estudiantes de nivel medio y/o universitario.

\section{Referencias}

Amichai-Hamburger, Y. \& Vinitzky, G. (2010). Social network use and personality. Computers in Human Behavior, 26, 12891295. http://dx.doi.org/10.1016/j.chb.2010.03.018

Amichai-Hamburger, Y., Wainapel, G., \& Fox, S. (2002). "On the Internet no one knows I'm an introvert": Extroversion, neuroticism, and
Internet interaction. CyberPsychology \& Behavior, 5(2), 125-128. http://dx.doi.org/10.1089/1094931027537705 07

Anderson, E. L., Steen, E., \& Stavropoulos, V. (2017). Internet use and problematic Internet use: A systematic review of longitudinal research trends in adolescence and emergent adulthood. International Journal of Adolescence and Youth, 22(4), 430-454. https://doi.org/10.1080/02673843.2016.12277 16

Blackwell, D., Leaman, C., Tramposch, R., Osborne, C., \& Liss, M. (2017). Extraversion, neuroticism, attachment style and fear of missing out as predictors of social media use and addiction. Personality and Individual Differences, 116, 69-72. https://doi.org/10.1016/j.paid.2017.04.039

Blease, C. R. (2015). Too many friends, too few likes? Evolutionary psychology and Facebook depression. Rev. Gen. Psychol., 19, 1-13 http://dx.doi.org/10.1037/gpr0000030

Burke, M., Marlow, C., \& Lento, T. (2010, April). Social network activity and social well-being. Paper presented at the conference on human factors in computing systems, Atlanta.

Castro Solano, A. (2000). Estilos de personalidad, objetivos de vida y satisfacción vital. Un estudio comparativo con adolescentes argentinos. Tesis Doctoral inédita. Universidad Complutense de Madrid. Facultad de Psicología.

Castro Solano, A., \& Casullo, M. M. (2001). Rasgos de personalidad, bienestar psicológico y rendimiento académico en adolescentes argentinos. Interdisciplinaria 18, 65-85.

Casullo, M., \& Castro Solano, A. (1999). Síntomas psicopatológicos en estudiantes adolescentes argentinos. Aportaciones del SCL90. Anuario de Investigaciones, VII, 147157.

Cohen, J. (1992). A power primer. Psychological Bulletin, 112(1), 155-159. https://doi.org/10.1037/0033-2909.112.1.155

Cosentino, A.C., \& Castro Solano, A. (2017). The High Five: Associations of the five positive factors with the Big Five and well-being. Frontiers in Psychology, 8, 1250. https://doi.org/10.3389/fpsyg.2017.01250 
Delfino, G., Sosa, F., Zubieta, E. (2017). Uso de internet en argentina: Género y edad como variables asociadas a la brecha digital. Investigación \& Desarrollo, 25(2), 100-123. http://dx.doi.org/10.14482/indes.25.2.10961

Derogatis L. (1977). SCL-90-R, administration, scoring and procedures manual for the $R$ (evised) version. Baltimore. Johns Hopkins University, School of Medicine.

Deters, F. G., \& Mehl, M. R. (2013). Does posting Facebook status updates increase or decrease loneliness? An online social networking experiment. Social Psychological \& Personality Science, 4, 579-586. http://dx.doi.org/10.1177/194855061246923

Diener, E., Emmons, R. A., Larsen, R. J., \& Griffin, S. (1985). The satisfaction with life scale. Journal of Personality Assessment, 49, 71-75. https://doi.org/10.1207/s15327752jpa4901_13

Diomidous, M., Chardalias, K., Magita, A., Koutonias, P., Panagiotopoulou, P., \& Mantas, J. (2016). Social and psychological effects of the Internet Use. Acta Inform. Med., 24 66-8. http://dx.doi.org/10.5455/aim.2016.24.66-68

Eftekhar, A., Fullwood, C., \& Morris, N. (2014). Capturing personality from Facebook photos and photo-related activities: How much exposure do you need? Computers in Human Behavior, 37, 162-170.

Fullwood, C., Nicholls, W., \& Makichi, R. (2014). We've got something for everyone: How individual differences predict different blogging motivations. New Media \& Society, $17 \quad$ (9), 1583-1600. https://doi.org/10.1177/1461444814530248

Gálvez-Nieto, J. L., Vera-Bachman, D., Cerda, C., \& Díaz, R. (2016). Escala de Victimización entre Adolescentes a través del Teléfono Móvil y de Internet: Estudio de validación de una versión abreviada en estudiantes chilenos. Revista Iberoamericana de Diagnóstico y Evaluación - e Avaliação Psicológica, 41(1), 16-27

Gilbert, G., \& Barton, H. (2013). The motivations and personality traits that influence Facebook usage. In A. Power \& G. Kirwan (Eds.), Cyberpsychology and new media: A thematic reader (pp. 26-37). Psychology Press, New York.

Goldberg, L. R., Johnson, J. A., Eber, H. W., Hogan, R., Ashton, M. C., Cloninger, C. R., et al. (2006). The international personality item pool and the future of public-domain personality measures. Journal of Research in Personality, 40(1), 84-96. https://doi.org/10.1016/j.jrp.2005.08.007

Guedes, E., Nardi, A. E., Guimarães, F. M. C. L., Machado, S., \& King, A. L. S. (2016). Social networking, a new online addiction: A review of Facebook and other addiction disorders. Med. Expr., 3, 1-6. doi:10.5935/medicalexpress.2016.01.01

Hamburger, Y. A., \& Ben-Artzi, E. (2000). The relationship between extraversion and neuroticism and the different uses of the Internet. Computers in Human Behavior, 16(4), 441-449. https://doi.org/10.1016/s07475632(00)00017-0

Huang, C. (2010). Internet use and psychological well-being: A meta-analysis. Cyberpsychol. Behav. Soc. Netw., 13(3), 241-9. https://doi.org/10.1089/cyber.2009.0217

Hughes, D. J., Rowe, M., Batey, M., \& Lee, A. (2012). A tale of two sites: Twitter vs. Facebook and the personality predictors of social media usage. Computers in Human Behavior, 28, 561-569. http://dx.doi.org/10.1016/j.chb.2011.11.001.

John, O. P., Donahue, E. M., \& Kentle, R. L. (1991). The Big Five Inventory - Versions $4 a$ and 54. Berkeley, CA: University of California, Berkeley, Institute of Personality and Social Research.

Kalpidou, M., Costin, D., \& Morris (2011). The relationship between Facebook and the wellbeing of undergraduate college students. Cyberpsychology, Behavior and Social Networking, 14(4), 183-9. https://doi.org/10.1089/cyber.2010.0061

Kayiş, A. R., Satici, S. A., Yilmaz, M. F., Şimşek, D., Ceyhan, E., \& Bakioğlu, F. (2016). Big five-personality trait and internet addiction: A meta-analytic review. Computers in Human Behavior, 63, 35-40. https://doi.org/10.1016/j.chb.2016.05.012.

Kim, J., \& Lee, J. E. R. (2011). The Facebook paths to happiness: Effects of the number of 
Facebook friends and self-presentation on subjective well-being. Cyberpsychology, Behavior, and Social Networking, 14, 359364. https://doi.org/10.1089/cyber.2010.0374

Krasnova, H., Wenninger, H., Widjaja, T., \& Buxmann, P. (2013). Envy on Facebook: A hidden threat to users' life satisfaction? Paper presented at the 11th International Conference on Wirtschaftsinformatik (WI), Leipzig, Germany

Kraut, R., Kiesler, S., Boneva, B., Cummings, J., Helgeson, V., \& Crawford, A. (2002). (2002) Internet paradox revisited. Journal of Social Issues, $\quad 58, \quad 49-74$. https://doi.org/10.1111/1540-4560.00248

Kraut, R., Patterson, M., Lundmark, V., Kiesler, S., Mukopadhyay, T., \& Scherlis, W. (1998). Internet paradox: A social technology that reduces social involvement and psychological well-being? American Psychologist, 53, 10171031. https://doi.org/10.1037//0003-066x.53.9.1017

Laconi, S., Tricard, N., \& Chabrol, H. (2015). Differences between specific and generalized problematic internet uses according to gender, age, time spent online and psychopathological symptoms. Computers in Human Behavior, 48 , 236-244. https://doi.org/10.1016/j.chb.2015.02.006

Marques, T. P., Marques Pinto, A., \& Alvarez, MJ. (2016). Estudo psicométrico da Escala de Avaliação dos Riscos e Oportunidades dos Jovens Utilizadores do Facebook. Revista Iberoamericana de Diagnóstico y Evaluación - e Avaliação Psicológica, 41(1), 145-158.

Nie, N., Hillygus, S., \& Erbring, L. (2002). Internet use, interpersonal relations and sociability: Findings from a detailed time diary study. In B. Wellman (Ed.), The Internet in Everyday Life (pp. 215-243). London: Blackwell Publishers.

Nie, P., Sousa-Poza, A., \& Nimrod, G. (2015). Internet use and subjective well-being in China. Social Indicators Research, 132, 489516. http://dx.doi.org/10.1007/s11205-015$1227-8$

Pérez, C. (2011). Técnicas de segmentación. Conceptos, herramientas y aplicaciones. Madrid: Gaceta Grupo Editorial.
Rubio-Hurtado, M.-J., \& Vilà-Baños, R. (2017). El análisis de conglomerados bietápico o en dos fases con SPSS. REIRE. Revista d'Innovació i Recerca en Educació, 10(1), 118-126. http://doi.org/10.1344/reire2017.10.11017

Ryan, T. \& Xenos, S. (2011). Who uses Facebook? An investigation into the relationship between the Big Five, shyness, narcissism, loneliness, and Facebook usage. Computers in Human Behavior, 27, 16581664. https://doi.org/10.1016/j.chb.2011.02.004

Seidman, G. (2013). Self-presentation and belonging on Facebook: How personality influences social media use and motivations. Personality and Individual Differences, 54(3), 402-407. https://doi.org/10.1016/j.paid.2012.10.009

Shaw, A. M., Timpano, K. R., Tran, T. B., \& Joormann, J. (2015). Correlates of Facebook usage patterns: The relationship between passive Facebook use, social anxiety symptoms, and brooding. Computers in Human Behavior, 48, 575-580 http://dx.doi.org/10.1016/ j.chb.2015.02.003.

Tausczik, Y. R., \& Pennebaker, J. W. (2010). The psychological meaning of words: LIWC and computerized text analysis methods. Journal of Language and Social Psychology, 29, 2454.

Tsitsika, A. K., Tzavela, E. C., Janikian, M., Olafsson, K., Iordache, A., Schoenmakers, T. M., et al. (2014). Online social networking in adolescence: Patterns of use in six European Countries and links with psychosocial functioning. Journal of Adolescent Health, 55(1), 141-147.

https://doi.org/10.1016/j.jadohealth.2013.11.010

Verduyn, P., Lee, D. S., Park, J., Shablack, H., Orvell, A., Bayer, J., et al. (2015). Passive Facebook usage undermines affective wellbeing: Experimental and longitudinal evidence. Journal of Experimental Psychology: General, 144(2), 480-488. https://doi.org/10.1037/xge0000057

Wilson, R. E., Gosling, S. D., \& Graham, L. T. (2012). A review of Facebook research in the social sciences. Perspectives on Psychological Science, $\quad 7(3), \quad 203-220$. https://doi.org/10.1177/1745691612442904 